\title{
Corrigendum \\ Antigen-43-mediated autoaggregation impairs motility in Escherichia coli
}

\author{
Glen C. Ulett, Richard I. Webb and Mark A. Schembri
}

Microbiology (2006), 152, 2101-2110

A printer's error led to an incorrect version of Fig. 1 being published. The correct version is shown below.

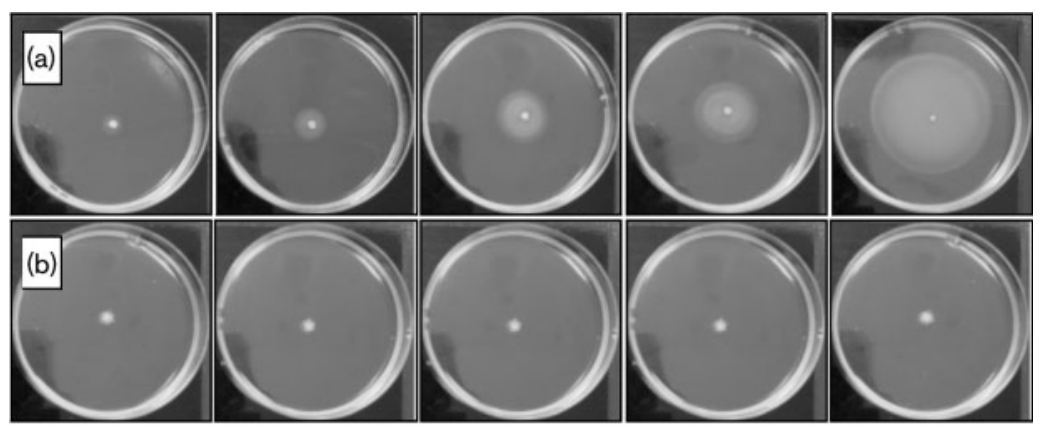

$16 \mathrm{~h}$

$18 \mathrm{~h}$

$20 \mathrm{~h}$

$22 \mathrm{~h}$

$24 \mathrm{~h}$

(c)

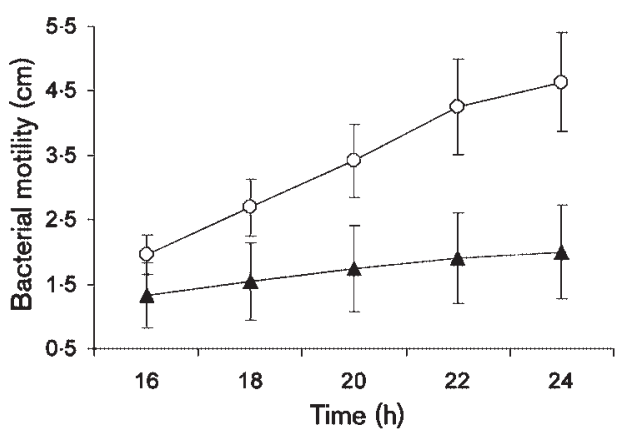

(d)

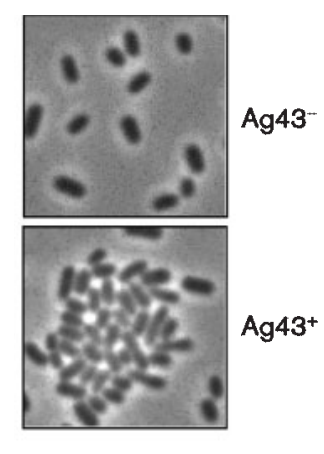

Fig. 1. Ag43 over-expressing OxyR-deficient E. coli MG1655 displays impaired swimming motility compared to the wild-type parent strain. Comparisons between strains were made using a semi-solid agar motility assay. MG1655 is highly motile (a) compared to strain MS641 (b) which lacks functional oxyR and therefore produces abundant amounts of the autoaggregator Ag43. The abatement of motility in MS641 is shown in (c: $\boldsymbol{\Delta}, \mathrm{MS641}$; O, MG1655), which is predicted to result from the proficient clumping activity of $\mathrm{Ag} 43$ (d). 